\title{
A short proof of affability for certain Cantor minimal $\mathbb{Z}^{2}$-systems
}

\author{
MATUI Hiroki
}

\begin{abstract}
We will show that any extension of a product of two Cantor minimal $\mathbb{Z}$-systems is affable in the sense of Giordano, Putnam and Skau.
\end{abstract}

\section{Introduction}

In this paper, we would like to investigate the orbit structure of certain minimal dynamical systems on a Cantor set. Giordano, Putnam and Skau proved that equivalence relations arising from $\mathbb{Z}$-actions are orbit equivalent to AF equivalence relations in GPS1. Moreover, they gave the classification for AF equivalence relations. In a recent paper GPS3, they continued their investigations and showed that equivalence relations arising from $\mathbb{Z}^{2}$-actions are again orbit equivalent to $\mathrm{AF}$ equivalence relations under a hypothesis involving the existence of cocycles. An equivalence relation which is orbit equivalent to an $\mathrm{AF}$ equivalence relation is said to be affable. A crucial ingredient of their proof was the absorption theorem given in GPS2. They needed, however, sufficiently many cocycles in order to construct an AF subequivalence relation to which the absorption theorem can be applied. The aim of this paper is to show that the existence of cocycles is not necessary for certain $\mathbb{Z}^{2}$-actions. We will give a short proof that the associated equivalence relations are orbit equivalent to $\mathrm{AF}$ equivalence relations, thus they are affable.

We recall some terminology that we shall use. Let $X$ be a Cantor set and let $\mathcal{R}$ be an étale equivalence relation on $X$. We define the $\mathcal{R}$-equivalence class $[x]_{\mathcal{R}}$ of $x \in X$ by $[x]_{\mathcal{R}}=\{y \in X$ : $(x, y) \in \mathcal{R}\}$. The equivalence relation $\mathcal{R}$ is said to be minimal, if $[x]_{\mathcal{R}}$ is dense in $X$ for every $x \in X$. Let $\varphi: G \rightarrow \operatorname{Homeo}(X)$ be a free action of a countable discrete group $G$, that is, $\varphi$ is a group homomorphism and $\varphi^{g}(x) \neq x$ for all $x \in X$ and $g \in G \backslash\{e\}$, where $e$ means the identity element. We put

$$
\mathcal{R}_{\varphi}=\left\{\left(x, \varphi^{g}(x)\right) \in X \times X: x \in X, g \in G\right\} .
$$

By transferring the product topology on $X \times G$ via the bijection $(x, g) \mapsto\left(x, \varphi^{g}(x)\right)$, we can topologize $\mathcal{R}_{\varphi}$. It is easily verified that $\mathcal{R}_{\varphi}$ becomes an étale equivalence relation. We call $(X, \varphi)$ a Cantor minimal $G$-system when $\mathcal{R}_{\varphi}$ is minimal. In this paper, we deal with only Cantor minimal $\mathbb{Z}$-systems and Cantor minimal $\mathbb{Z}^{2}$-systems.

Let $(X, \varphi)$ and $(Y, \psi)$ be two Cantor minimal $\mathbb{Z}^{2}$-systems. We say that $\pi:(Y, \psi) \rightarrow(X, \varphi)$ is a factor map when $\pi: Y \rightarrow X$ is a continuous map and $\pi \circ \psi^{a}=\varphi^{a} \circ \pi$ for all $a \in \mathbb{Z}^{2}$. The system $(Y, \psi)$ is called an extension of $(X, \varphi)$. Our main theorem asserts that $\mathcal{R}_{\psi}$ is affable, if $(X, \varphi)$ is conjugate to a product of two Cantor minimal $\mathbb{Z}$-systems. Suppose that $(X, \varphi)$ is conjugate to the product of two Cantor minimal $\mathbb{Z}$-systems $\left(X_{1}, \varphi_{1}\right)$ and $\left(X_{2}, \varphi_{2}\right)$. From GPS1 Theorem 2.3] we can see that $\mathcal{R}_{\varphi_{1}}$ and $\mathcal{R}_{\varphi_{2}}$ are affable. Since a product of AF equivalence relations is also AF, it is easily checked that $\mathcal{R}_{\varphi}$ is affable. But, it looks impossible to mimic this simple argument in the case of the extension $(Y, \psi)$, because $(Y, \psi)$ itself is not a product. 
We will instead construct a 'nice' AF subequivalence relation of $\mathcal{R}_{\varphi}$ and apply the absorption theorem to this relation.

\section{Products of Cantor minimal $\mathbb{Z}$-systems}

Throughout this section, let $B_{i}=\left(V_{i}, E_{i}\right)$ be simple properly ordered Bratteli diagrams for $i=1,2$. For each $i=1,2, V_{i}$ and $E_{i}$ can be written as a countable disjoint union of non-empty finite sets:

$$
V_{i}=V_{i, 0} \cup V_{i, 1} \cup V_{i, 2} \cup \ldots \text { and } E_{i}=E_{i, 1} \cup E_{i, 2} \cup E_{i, 3} \cup \ldots
$$

with the source map $s: E_{i, n} \rightarrow V_{i, n-1}$ and the range map $r: E_{i, n} \rightarrow V_{i, n}$. Without loss of generality, we may assume that all two vertices in consecutive levels are connected by more than three edges. We write the infinite path space associated with $B_{i}$ by $X_{i}$ for each $i=1,2$. Let $p_{i}$ be the unique maximal infinite path of $X_{i}$ and let $\varphi_{i} \in \operatorname{Homeo}\left(X_{i}\right)$ be the Bratteli-Vershik transformation on $X_{i}$ (see [HPS]). It is well known that $\left(X_{i}, \varphi_{i}\right)$ is a Cantor minimal $\mathbb{Z}$-system.

Set $X=X_{1} \times X_{2}$. Let $\varphi: \mathbb{Z}^{2} \rightarrow \operatorname{Homeo}(X)$ be the $\mathbb{Z}^{2}$-action on $X$ induced by $\varphi_{1} \times$ id and id $\times \varphi_{2}$. Then, $(X, \varphi)$ is a Cantor minimal $\mathbb{Z}^{2}$-system.

For each $n \in \mathbb{N}$, we put

$$
\mathcal{R}_{n}=\left\{\left(\left(x_{1}, x_{2}\right),\left(y_{1}, y_{2}\right)\right) \in X \times X: x_{i, m}=y_{i, m} \text { for } i=1,2 \text { and } m>n\right\},
$$

where $x_{i, m}, y_{i, m} \in E_{i, m}$ mean the $m$-th coordinate of the infinite paths $x_{i}, y_{i} \in X_{i}$. It is not hard to see that $\mathcal{R}_{n}$ is a compact open subequivalence relation of $\mathcal{R}_{\varphi}$ with the relative topology from $\mathcal{R}_{\varphi}$. Therefore

$$
\mathcal{R}=\bigcup_{n \in \mathbb{N}} \mathcal{R}_{n}
$$

is an AF subequivalence relation of $\mathcal{R}_{\varphi}$. Note that $\mathcal{R}$ is minimal because $B_{1}$ and $B_{2}$ are simple.

For $i, j=0,1$ and $n \in \mathbb{N}$, we define continuous functions $\lambda_{n}^{i j}: X \rightarrow\{0,1\}$ inductively as follows. Let $\left(x_{1}, x_{2}\right) \in X$. We denote the $n$-th coordinate of $x_{i}$ by $x_{i, n} \in E_{i . n}$. At first, put

$$
\begin{aligned}
& \lambda_{1}^{00}\left(x_{1}, x_{2}\right)= \begin{cases}1 & x_{1,1} \text { is maximal } \\
0 & \text { otherwise },\end{cases} \\
& \lambda_{1}^{01}\left(x_{1}, x_{2}\right)= \begin{cases}1 & x_{2,1} \text { is minimal } \\
0 & \text { otherwise },\end{cases} \\
& \lambda_{1}^{11}\left(x_{1}, x_{2}\right)= \begin{cases}1 & x_{1,1} \text { is minimal } \\
0 & \text { otherwise }\end{cases}
\end{aligned}
$$

and

$$
\lambda_{1}^{10}\left(x_{1}, x_{2}\right)= \begin{cases}1 & x_{2,1} \text { is maximal } \\ 0 & \text { otherwise }\end{cases}
$$

Then, for $n \geq 2$, we define $\lambda_{n}^{i j}$ by

$$
\lambda_{n}^{00}\left(x_{1}, x_{2}\right)= \begin{cases}\lambda_{n-1}^{00}\left(x_{1}, x_{2}\right) & x_{1, n} \text { is maximal and } x_{2, n} \text { is maximal } \\ 1 & x_{1, n} \text { is maximal and } x_{2, n} \text { is not maximal } \\ 0 & \text { otherwise }\end{cases}
$$




$$
\begin{aligned}
& \lambda_{n}^{01}\left(x_{1}, x_{2}\right)= \begin{cases}\lambda_{n-1}^{01}\left(x_{1}, x_{2}\right) & x_{1, n} \text { is maximal and } x_{2, n} \text { is minimal } \\
1 & x_{1, n} \text { is not maximal and } x_{2, n} \text { is minimal } \\
0 & \text { otherwise }\end{cases} \\
& \lambda_{n}^{11}\left(x_{1}, x_{2}\right)= \begin{cases}\lambda_{n-1}^{11}\left(x_{1}, x_{2}\right) & x_{1, n} \text { is minimal and } x_{2, n} \text { is minimal } \\
1 & x_{1, n} \text { is minimal and } x_{2, n} \text { is not minimal } \\
0 & \text { otherwise }\end{cases}
\end{aligned}
$$

and

$$
\lambda_{n}^{10}\left(x_{1}, x_{2}\right)= \begin{cases}\lambda_{n-1}^{10}\left(x_{1}, x_{2}\right) & x_{1, n} \text { is minimal and } x_{2, n} \text { is maximal } \\ 1 & x_{1, n} \text { is not minimal and } x_{2, n} \text { is maximal } \\ 0 & \text { otherwise }\end{cases}
$$

It is easily checked that $\lambda_{n}^{i j}$ is well-defined and continuous.

The following is an immediate consequence of the definition of $\lambda_{n}^{i j}$.

Lemma 2.1. Let $(i, j) \in\{0,1\}^{2}$. For $\left(\left(x_{1}, x_{2}\right),\left(y_{1}, y_{2}\right)\right) \in \mathcal{R}_{n}$, if

$$
\lambda_{n}^{i j}\left(x_{1}, x_{2}\right)=\lambda_{n}^{i j}\left(y_{1}, y_{2}\right)
$$

then we have

$$
\lambda_{m}^{i j}\left(x_{1}, x_{2}\right)=\lambda_{m}^{i j}\left(y_{1}, y_{2}\right)
$$

for all $m>n$.

For every $n \in \mathbb{N}$, we define a subset $\mathcal{R}_{n}^{\prime}$ of $\mathcal{R}_{n}$ by

$$
\mathcal{R}_{n}^{\prime}=\left\{\left(\left(x_{1}, x_{2}\right),\left(y_{1}, y_{2}\right)\right) \in \mathcal{R}_{n}: \lambda_{n}^{i j}\left(x_{1}, x_{2}\right)=\lambda_{n}^{i j}\left(y_{1}, y_{2}\right) \text { for all } i, j=0,1\right\} .
$$

Lemma 2.2. For every $n \in \mathbb{N}, \mathcal{R}_{n}^{\prime}$ is a compact open subequivalence relation of $\mathcal{R}_{n}$, and $\mathcal{R}_{n}^{\prime}$ is contained in $\mathcal{R}_{n+1}^{\prime}$.

Proof. It is obvious that $\mathcal{R}_{n}^{\prime}$ is a subequivalence relation of $\mathcal{R}_{n}$. Since $\lambda_{n}^{i j}$ is continuous, $\mathcal{R}_{n}^{\prime}$ is compact and open. From the lemma above we can see $\mathcal{R}_{n}^{\prime} \subset \mathcal{R}_{n+1}^{\prime}$.

Define

$$
\mathcal{R}^{\prime}=\bigcup_{n \in \mathbb{N}} \mathcal{R}_{n}^{\prime}
$$

By the lemma above, $\mathcal{R}^{\prime}$ is an $\mathrm{AF}$ equivalence relation on $X$.

Lemma 2.3. Let $\left(\left(x_{1}, x_{2}\right),\left(y_{1}, y_{2}\right)\right) \in \mathcal{R}$.

(1) If $x_{1}$ is not in $\left\{\varphi_{1}^{n}\left(p_{1}\right): n \in \mathbb{Z}\right\}$, then $\left(\left(x_{1}, x_{2}\right),\left(y_{1}, y_{2}\right)\right) \in \mathcal{R}^{\prime}$.

(2) If $x_{2}$ is not in $\left\{\varphi_{2}^{n}\left(p_{2}\right): n \in \mathbb{Z}\right\}$, then $\left(\left(x_{1}, x_{2}\right),\left(y_{1}, y_{2}\right)\right) \in \mathcal{R}^{\prime}$.

Proof. It suffices to show (1). There exists $n \in \mathbb{N}$ such that $\left(\left(x_{1}, x_{2}\right),\left(y_{1}, y_{2}\right)\right) \in \mathcal{R}_{n}$. We can find a natural number $m>n$ such that $x_{1, m}$ is not maximal. Then, $\lambda_{m}^{00}\left(x_{1}, x_{2}\right)$ equals zero. From $x_{1, m}=y_{1, m}$, we get $\lambda_{m}^{00}\left(x_{1}, x_{2}\right)=\lambda_{m}^{00}\left(y_{1}, y_{2}\right)=0$. It is easy to see that $\lambda_{m}^{01}\left(x_{1}, x_{2}\right)$ depends only on $x_{2, m}$, and so we have $\lambda_{m}^{01}\left(x_{1}, x_{2}\right)=\lambda_{m}^{01}\left(y_{1}, y_{2}\right)$.

We can find a natural number $l>n$ such that $x_{1, l}$ is not minimal. It is clear that $\lambda_{l}^{11}\left(x_{1}, x_{2}\right)=$ 0 and $\lambda_{l}^{10}\left(x_{1}, x_{2}\right)$ depends only on $x_{2, l}$. In a similar fashion to the preceding paragraph, we get $\lambda_{l}^{11}\left(x_{1}, x_{2}\right)=\lambda_{l}^{11}\left(y_{1}, y_{2}\right)$ and $\lambda_{l}^{10}\left(x_{1}, x_{2}\right)=\lambda_{l}^{10}\left(y_{1}, y_{2}\right)$.

By virtue of Lemma 2.1 we can conclude that $\left(\left(x_{1}, x_{2}\right),\left(y_{1}, y_{2}\right)\right)$ is in $\mathcal{R}_{k}^{\prime}$, where $k$ is the maximum of $m$ and $l$. 
Put $p=\left(p_{1}, p_{2}\right) \in X$. The above lemma tells us that the four $\mathcal{R}$-orbits $[p]_{\mathcal{R}},\left[\varphi^{(1,0)}(p)\right]_{\mathcal{R}}$, $\left[\varphi^{(0,1)}(p)\right]_{\mathcal{R}}$ and $\left[\varphi^{(1,1)}(p)\right]_{\mathcal{R}}$ may split in $\mathcal{R}^{\prime}$, but the other $\mathcal{R}$-orbits do not split in $\mathcal{R}^{\prime}$.

Lemma 2.4. The equivalence relation $\mathcal{R}^{\prime}$ is minimal.

Proof. Let $\left(x_{1}, x_{2}\right) \in X$. It suffices to show that $\left[\left(x_{1}, x_{2}\right)\right]_{\mathcal{R}^{\prime}}$ is dense in $X$. If $x_{1}$ does not belong to $\left\{\varphi_{1}^{n}\left(p_{1}\right): n \in \mathbb{Z}\right\}$ or $x_{2}$ does not belong to $\left\{\varphi_{2}^{n}\left(p_{2}\right): n \in \mathbb{Z}\right\}$, then we have nothing to do, because the $\mathcal{R}^{\prime}$-orbit of $\left(x_{1}, x_{2}\right)$ is equal to the $\mathcal{R}$-orbit of it. Suppose that $\left(x_{1}, x_{2}\right)$ is in $\left\{\varphi^{a}(p): a \in \mathbb{Z}^{2}\right\}$. Without loss of generality, we may assume that $\left(x_{1}, x_{2}\right)$ belongs to $[p]_{\mathcal{R}}$. Take finite paths $\left(e_{1,1}, e_{1,2}, \ldots, e_{1, n}\right)$ in $B_{1}$ and $\left(e_{2,1}, e_{2,2}, \ldots, e_{2, n}\right)$ in $B_{2}$. Thus $e_{i, k} \in E_{i, k}$ and $r\left(e_{i, k}\right)=s\left(e_{i, k+1}\right)$. We can find $m>n+2$ such that both $x_{1, m}$ and $x_{2, m}$ are maximal. It follows that $\lambda_{m}^{01}\left(x_{1}, x_{2}\right)=0, \lambda_{m}^{11}\left(x_{1}, x_{2}\right)=0$ and $\lambda_{m}^{10}\left(x_{1}, x_{2}\right)=1$. We have two possibilities: $\lambda_{m}^{00}\left(x_{1}, x_{2}\right)=0$ or 1 .

Let us consider the case that $\lambda_{m}^{00}\left(x_{1}, x_{2}\right)$ is one. We can find edges $e_{i, k} \in E_{i, k}$ for $i=1,2$ and $k=n+1, n+2, \ldots, m-1$ such that the following are satisfied.

- $r\left(e_{i, k}\right)=s\left(e_{i, k+1}\right)$ and $r\left(e_{i, m-1}\right)=s\left(x_{i, m}\right)$ for all $i=1,2$ and $k=n, n+1, \ldots, m-2$.

- $e_{1, m-1}$ is maximal and $e_{2, m-1}$ is not maximal.

Put

$$
x_{i}^{\prime}=\left(e_{i, 1}, e_{i, 2}, \ldots, e_{i, n}, e_{i, n+1}, \ldots, e_{i, m-1}, x_{i, m}, x_{i, m+1} \ldots\right) \in X_{i}
$$

for each $i=1,2$. Then it is clear that $\left(\left(x_{1}, x_{2}\right),\left(x_{1}^{\prime}, x_{2}^{\prime}\right)\right) \in \mathcal{R}_{m}$. Moreover, it is not hard to see $\lambda_{m}^{00}\left(x_{1}^{\prime}, x_{2}^{\prime}\right)=1, \lambda_{m}^{01}\left(x_{1}^{\prime}, x_{2}^{\prime}\right)=0, \lambda_{m}^{11}\left(x_{1}^{\prime}, x_{2}^{\prime}\right)=0$ and $\lambda_{m}^{10}\left(x_{1}^{\prime}, x_{2}^{\prime}\right)=1$. Therefore we get $\left(\left(x_{1}, x_{2}\right),\left(x_{1}^{\prime}, x_{2}^{\prime}\right)\right) \in \mathcal{R}_{m}^{\prime}$.

Suppose that $\lambda_{m}^{00}\left(x_{1}, x_{2}\right)$ is zero. In this case we choose the edges $e_{i, k} \in E_{i, k}$ so that the following are satisfied.

- $r\left(e_{i, k}\right)=s\left(e_{i, k+1}\right)$ and $r\left(e_{i, m-1}\right)=s\left(x_{i, m}\right)$ for all $i=1,2$ and $k=n, n+1, \ldots, m-2$.

- $e_{1, m-1}$ is not maximal.

Then, we can again obtain $\left(\left(x_{1}, x_{2}\right),\left(x_{1}^{\prime}, x_{2}^{\prime}\right)\right) \in \mathcal{R}_{m}^{\prime}$. Hence we can conclude that the $\mathcal{R}^{\prime}$-orbit of $\left(x_{1}, x_{2}\right)$ is dense in $X$.

Lemma 2.5. For every $m \in \mathbb{N} \backslash\{1\}$ we have the following.

(1) $\lim _{n \rightarrow \infty}\left(\lambda_{n}^{00} \times \lambda_{n}^{01} \times \lambda_{n}^{11} \times \lambda_{n}^{10}\right)\left(p_{1}, \varphi_{2}^{1-m}\left(p_{2}\right)\right)=(1,0,0,1)$.

(2) $\lim _{n \rightarrow \infty}\left(\lambda_{n}^{00} \times \lambda_{n}^{01} \times \lambda_{n}^{11} \times \lambda_{n}^{10}\right)\left(\varphi_{1}\left(p_{1}\right), \varphi_{2}^{1-m}\left(p_{2}\right)\right)=(0,0,1,0)$.

(3) $\lim _{n \rightarrow \infty}\left(\lambda_{n}^{00} \times \lambda_{n}^{01} \times \lambda_{n}^{11} \times \lambda_{n}^{10}\right)\left(p_{1}, \varphi_{2}^{m}\left(p_{2}\right)\right)=(1,0,0,0)$.

(4) $\lim _{n \rightarrow \infty}\left(\lambda_{n}^{00} \times \lambda_{n}^{01} \times \lambda_{n}^{11} \times \lambda_{n}^{10}\right)\left(\varphi_{1}\left(p_{1}\right), \varphi_{2}^{m}\left(p_{2}\right)\right)=(0,1,1,0)$.

(5) $\lim _{n \rightarrow \infty}\left(\lambda_{n}^{00} \times \lambda_{n}^{01} \times \lambda_{n}^{11} \times \lambda_{n}^{10}\right)\left(\varphi_{1}^{1-m}\left(p_{1}\right), p_{2}\right)=(0,0,0,1)$.

(6) $\lim _{n \rightarrow \infty}\left(\lambda_{n}^{00} \times \lambda_{n}^{01} \times \lambda_{n}^{11} \times \lambda_{n}^{10}\right)\left(\varphi_{1}^{1-m}\left(p_{1}\right), \varphi_{2}\left(p_{2}\right)\right)=(1,1,0,0)$.

(7) $\lim _{n \rightarrow \infty}\left(\lambda_{n}^{00} \times \lambda_{n}^{01} \times \lambda_{n}^{11} \times \lambda_{n}^{10}\right)\left(\varphi_{1}^{m}\left(p_{1}\right), p_{2}\right)=(0,0,1,1)$.

(8) $\lim _{n \rightarrow \infty}\left(\lambda_{n}^{00} \times \lambda_{n}^{01} \times \lambda_{n}^{11} \times \lambda_{n}^{10}\right)\left(\varphi_{1}^{m}\left(p_{1}\right), \varphi_{2}\left(p_{2}\right)\right)=(0,1,0,0)$.

Proof. Straightforward computation. 
Take a clopen subset $U_{i} \subset X_{i}$ which does not contain $p_{i}$ and $\varphi_{i}\left(p_{i}\right)$ for each $i=1,2$. Put

$$
B=\left(\left\{p_{1}\right\} \times U_{2}\right) \cup\left(U_{1} \times\left\{p_{2}\right\}\right)
$$

and

$$
B^{*}=\left(\left\{\varphi_{1}\left(p_{1}\right)\right\} \times U_{2}\right) \cup\left(U_{1} \times\left\{\varphi_{2}\left(p_{2}\right)\right\}\right) .
$$

Lemma 2.6. Both $B$ and $B^{*}$ are closed $\mathcal{R}^{\prime}$-étale thin subsets.

Proof. It suffices to show the statement for $B$. Suppose that $\left(\left(x_{1}, x_{2}\right),\left(y_{1}, y_{2}\right)\right)$ is in $\mathcal{R}^{\prime} \cap(B \times B)$. Without loss of generality, we may assume $x_{1}=p_{1}$. Suppose $y_{2}=p_{2}$. Then $x_{2}$ must be $\varphi_{2}^{1-m}\left(p_{2}\right)$ for some $m \in \mathbb{N}$, and $m$ is not one because $p_{2}$ is not in $U_{2}$. Similarly $y_{1}$ must be $\varphi_{1}^{1-l}\left(p_{1}\right)$ for some $l \in \mathbb{N} \backslash\{1\}$. But $\left(\left(p_{1}, \varphi_{1}^{1-m}\left(p_{2}\right)\right),\left(\varphi_{1}^{1-l}\left(p_{1}\right), p_{2}\right)\right)$ never be in $\mathcal{R}^{\prime}$ by the lemma above. Hence we have $y_{1}=p_{1}$. Thus $\left(\left(x_{1}, x_{2}\right),\left(y_{1}, y_{2}\right)\right)$ is equal to $\left(\left(p_{1}, x_{2}\right),\left(p_{1}, \varphi_{2}^{m}\left(x_{2}\right)\right)\right)$ for some $m \in \mathbb{Z}$. Define

$$
V=\left\{((a, b),(c, d)) \in \mathcal{R}^{\prime}: a=c, d=\varphi_{2}^{m}(b) \text { and } b, d \in U_{2}\right\} .
$$

Then $V$ is a clopen neighborhood of $\left(\left(p_{1}, x_{2}\right),\left(p_{1}, \varphi_{2}^{m}\left(x_{2}\right)\right)\right)$ in $\mathcal{R}^{\prime}$. For $((a, b),(c, d)) \in V$, it is obvious that $(a, b) \in B$ if and only if $(c, d) \in B$, which implies that $B$ is étale.

We would like to show that a probability measure on $X=X_{1} \times X_{2}$ is $\mathcal{R}$-invariant if and only if it is $\mathcal{R}^{\prime}$-invariant. If this is shown, thinness of $B$ easily follows. But, except for countably many $\left(x_{1}, x_{2}\right)$ 's, the equivalence class $\left[\left(x_{1}, x_{2}\right)\right]_{\mathcal{R}}$ is equal to $\left[\left(x_{1}, x_{2}\right)\right]_{\mathcal{R}^{\prime}}$. Since every invariant measure is nonatomic, we can finish the proof.

Lemma 2.7. We have $\mathcal{R}^{\prime} \cap\left(B \times B^{*}\right)=\emptyset$.

Proof. Suppose that $\left(\left(x_{1}, x_{2}\right),\left(y_{1}, y_{2}\right)\right)$ is contained in $\mathcal{R}^{\prime} \cap\left(B \times B^{*}\right)$. Without loss of generality, we may assume $x_{1}=p_{1}$. Then $y_{1}$ never be $\varphi_{1}\left(x_{1}\right)$, because $\left(\left(p_{1}, x_{2}\right),\left(\varphi_{1}\left(p_{1}\right), y_{2}\right)\right)$ does not belong to $\mathcal{R}$. It follows that $y_{2}=\varphi_{2}\left(p_{2}\right)$ and $\left(\left(x_{1}, x_{2}\right),\left(y_{1}, y_{2}\right)\right)=\left(\left(p_{1}, \varphi_{2}^{m}\left(p_{2}\right)\right),\left(\varphi_{1}^{1-l}\left(p_{1}\right), \varphi_{2}\left(p_{2}\right)\right)\right)$ for some $m, l \in \mathbb{N} \backslash\{1\}$. This pair, however, never belongs to $\mathcal{R}^{\prime}$ by virtue of Lemma 2.5], which completes the proof.

We define a homeomorphism $\beta: B \rightarrow B^{*}$ as follows. For $\left(p_{1}, x_{2}\right) \in\left\{p_{1}\right\} \times U_{2}$, we put $\beta\left(p_{1}, x_{2}\right)=\left(\varphi_{1}\left(p_{1}\right), x_{2}\right)$. For $\left(x_{1}, p_{2}\right) \in U_{1} \times\left\{p_{2}\right\}$, we put $\beta\left(x_{1}, p_{2}\right)=\left(x_{1}, \varphi_{2}\left(p_{2}\right)\right)$.

Lemma 2.8. The homeomorphism $\beta: B \rightarrow B^{*}$ induces an isomorphism between $\mathcal{R}^{\prime} \cap(B \times B)$ and $\mathcal{R}^{\prime} \cap\left(B^{*} \times B^{*}\right)$.

Proof. Since the topology of $\mathcal{R}^{\prime} \cap(B \times B)$ and $\mathcal{R}^{\prime} \cap\left(B^{*} \times B^{*}\right)$ is inherited from $\mathcal{R}$, it suffices to show that $\beta$ is a well-defined bijection between $\mathcal{R}^{\prime} \cap(B \times B)$ and $\mathcal{R}^{\prime} \cap\left(B^{*} \times B^{*}\right)$. Let $\left(\left(x_{1}, x_{2}\right),\left(y_{1}, y_{2}\right)\right) \in \mathcal{R}^{\prime} \cap(B \times B)$. Without loss of generality, we may assume that $x_{1}=p_{1}$ and $x_{2} \in U_{2}$. By the proof of Lemma 2.6] we get $y_{1}=p_{1}$ and $y_{2} \in U_{2}$. It follows that $\beta\left(p_{1}, x_{2}\right)=$ $\left(\varphi_{1}\left(p_{1}\right), x_{2}\right)$ and $\beta\left(p_{1}, y_{2}\right)=\left(\varphi_{1}\left(p_{1}\right), y_{2}\right)$. If $x_{2}$ does not belong to $\left\{\varphi_{2}^{n}\left(p_{2}\right): n \in \mathbb{Z}\right\}$, then the $\mathcal{R}^{\prime}$-orbit of $\left(\varphi_{1}\left(p_{1}\right), x_{2}\right)$ is equal to the $\mathcal{R}$-orbit of it. Hence we have $\left(\left(\varphi_{1}\left(p_{1}\right), x_{2}\right),\left(\varphi_{1}\left(p_{1}\right), y_{2}\right)\right) \in$ $\mathcal{R}^{\prime} \cap\left(B^{*} \times B^{*}\right)$.

Suppose that $x_{2}$ and $y_{2}$ belong to $\left\{\varphi_{2}^{n}\left(p_{2}\right): n \in \mathbb{Z}\right\}$. Since $\left(\left(p, x_{2}\right),\left(p, y_{2}\right)\right) \in \mathcal{R}^{\prime} \subset \mathcal{R}$, we have two possibilities: both $x_{2}$ and $y_{2}$ belong to $\left\{\varphi_{2}^{1-n}\left(p_{2}\right): n \in \mathbb{N}\right\}$, or both $x_{2}$ and $y_{2}$ belong to $\left\{\varphi_{2}^{n}\left(p_{2}\right): n \in \mathbb{N}\right\}$. Without loss of generality, we may assume the latter. Thus, $x_{2}=\varphi_{2}^{n}\left(p_{2}\right)$ and $y_{2}=\varphi_{2}^{m}\left(p_{2}\right)$ for some $n, m \in \mathbb{N}$. Because $x_{2}$ and $y_{2}$ is in $U_{2}, n$ and $m$ are greater than one. It follows from Lemma 2.5 that $\left(\left(\varphi_{1}\left(p_{1}\right), \varphi_{2}^{n}\left(p_{2}\right)\right),\left(\varphi_{1}\left(p_{1}\right), \varphi_{2}^{m}\left(p_{2}\right)\right)\right)$ belongs to $\mathcal{R}^{\prime}$. The proof is completed.

Lemma 2.9. Let $\tilde{\mathcal{R}}$ be the equivalence relation generated by $\mathcal{R}^{\prime}$ and the graph of $\beta$. Then $\mathcal{R}_{\varphi}$ is generated by $\tilde{\mathcal{R}}$ and $\left(p, \varphi^{(0,1)}(p)\right),\left(\varphi^{(0,1)}(p), \varphi^{(1,1)}(p)\right)$ and $\left(\varphi^{(1,1)}(p), \varphi^{(1,0)}(p)\right)$. 
Proof. Evidently $\mathcal{R}_{\varphi}$ is generated by $\mathcal{R}$ and the graph of $\beta$. As mentioned before, if $\left(x_{1}, x_{2}\right)$ is not contained in the $\mathcal{R}_{\varphi}$-orbit of $p=\left(p_{1}, p_{2}\right)$, then its $\mathcal{R}$-orbit agrees with its $\mathcal{R}^{\prime}$-orbit. It follows that the $\tilde{\mathcal{R}}$-orbit of $\left(x_{1}, x_{2}\right)$ agrees with the $\mathcal{R}_{\varphi}$-orbit of it.

Let us consider $[p]_{\mathcal{R}_{\varphi}}$. Notice that it splits into four orbits in $\mathcal{R}$, namely the $\mathcal{R}$-orbits of $p, \varphi^{(0,1)}(p), \varphi^{(1,0)}(p)$ and $\varphi^{(1,1)}(p)$. From Lemma 2.5 we can see that these orbits split into eight orbits in $\mathcal{R}^{\prime}$, namely the $\mathcal{R}^{\prime}$-orbits of $p, \varphi^{(-1,0)}(p), \varphi^{(0,1)}(p), \varphi^{(0,2)}(p), \varphi^{(1,0)}(p), \varphi^{(1,-1)}(p)$, $\varphi^{(1,1)}(p)$ and $\varphi^{(2,1)}(p)$. It can be easily seen that

$$
\begin{aligned}
{[p]_{\tilde{\mathcal{R}}} } & =[p]_{\mathcal{R}^{\prime}} \cup\left[\varphi^{(1,-1)}(p)\right]_{\mathcal{R}^{\prime}}, \\
{\left[\varphi^{(0,1)}(p)\right]_{\tilde{\mathcal{R}}} } & =\left[\varphi^{(0,1)}(p)\right]_{\mathcal{R}^{\prime}} \cup\left[\varphi^{(-1,0)}(p)\right]_{\mathcal{R}^{\prime}}, \\
{\left[\varphi^{(1,0)}(p)\right]_{\tilde{\mathcal{R}}} } & =\left[\varphi^{(1,0)}(p)\right]_{\mathcal{R}^{\prime}} \cup\left[\varphi^{(2,1)}(p)\right]_{\mathcal{R}^{\prime}}
\end{aligned}
$$

and

$$
\left[\varphi^{(1,1)}(p)\right]_{\tilde{\mathcal{R}}}=\left[\varphi^{(1,1)}(p)\right]_{\mathcal{R}^{\prime}} \cup\left[\varphi^{(0,2)}(p)\right]_{\mathcal{R}^{\prime}}
$$

Therefore, by glueing the $\tilde{\mathcal{R}}$-orbits of $p, \varphi^{(0,1)}(p), \varphi^{(1,0)}(p)$ and $\varphi^{(1,1)}(p)$, we can recover the equivalence relation $\mathcal{R}_{\varphi}$.

By [HPS, Theorem 4.6], every minimal homeomorphism on the Cantor set is conjugate to a Bratteli-Vershik transformation on a simple properly ordered Bratteli diagram. Hence we can summarize the results obtained in this section as follows.

Theorem 2.10. Let $\left(X_{1}, \varphi_{1}\right)$ and $\left(X_{2}, \varphi_{2}\right)$ be two Cantor minimal $\mathbb{Z}$-systems and let $p_{1} \in X_{1}$ and $p_{2} \in X_{2}$. Take clopen subsets $U_{1} \subset X_{1}$ and $U_{2} \subset X_{2}$ so that $p_{i}$ and $\varphi_{i}\left(p_{i}\right)$ do not belong to $U_{i}$ for each $i=1,2$. Put $B=\left(\left\{p_{1}\right\} \times U_{2}\right) \cup\left(U_{1} \times\left\{p_{2}\right\}\right)$ and $B^{*}=\left(\left\{\varphi_{1}\left(p_{1}\right)\right\} \times U_{2}\right) \cup\left(U_{1} \times\left\{\varphi_{2}\left(p_{2}\right)\right\}\right)$. Define $\beta: B \rightarrow B^{*}$ by $\beta\left(p_{1}, x_{2}\right)=\left(\varphi_{1}\left(p_{1}\right), x_{2}\right)$ and $\beta\left(x_{1}, p_{2}\right)=\left(x_{1}, \varphi_{2}\left(p_{2}\right)\right)$. Let $\varphi$ be the $\mathbb{Z}^{2}$-action on $X=X_{1} \times X_{2}$ induced by $\varphi_{1} \times$ id and id $\times \varphi_{2}$. Put $p=\left(p_{1}, p_{2}\right)$.

Then we can find a subequivalence relation $\mathcal{R}^{\prime} \subset \mathcal{R}_{\varphi}$ such that the following are satisfied.

(1) $\mathcal{R}^{\prime}$ is a minimal AF equivalence relation, where the topology is given by $\mathcal{R}_{\varphi}$.

(2) Both $B$ and $B^{*}$ are closed $\mathcal{R}^{\prime}$-étale thin subsets.

(3) $\mathcal{R}^{\prime} \cap\left(B \times B^{*}\right)$ is empty.

(4) $\beta: B \rightarrow B^{*}$ induces an isomorphism between $\mathcal{R}^{\prime} \cap(B \times B)$ and $\mathcal{R}^{\prime} \cap\left(B^{*} \times B^{*}\right)$.

(5) The equivalence relation $\mathcal{R}_{\varphi}$ is generated by $\mathcal{R}^{\prime}$, the graph of $\beta$ and

$$
\left\{\left(p, \varphi^{(0,1)}(p)\right),\left(\varphi^{(0,1)}(p), \varphi^{(1,1)}(p)\right)\left(\varphi^{(1,1)}(p), \varphi^{(1,0)}(p)\right)\right\}
$$

\section{The main result}

Let $(X, \varphi)$ and $(Y, \psi)$ be two Cantor minimal $\mathbb{Z}^{2}$-systems and let $\pi:(Y, \psi) \rightarrow(X, \varphi)$ be a factor map.

Lemma 3.1. Suppose that $\mathcal{R}$ is an open subequivalence relation of $\mathcal{R}_{\varphi}$. For

$$
\mathcal{S}=\left\{\left(y, y^{\prime}\right) \in \mathcal{R}_{\psi}:\left(\pi(y), \pi\left(y^{\prime}\right)\right) \in \mathcal{R}\right\},
$$

we have the following. 
(1) If $\mathcal{R}$ is compact and open, then $\mathcal{S}$ is also compact and open.

(2) If $\mathcal{R}$ is $A F$, then $\mathcal{S}$ is also $A F$.

Proof. (2) follows immediately from (1). Suppose that $\mathcal{R}$ is compact and open. Since $\pi \times \pi$ : $\mathcal{R}_{\psi} \rightarrow \mathcal{R}_{\varphi}$ is proper and continuous, we can see that $\mathcal{S}=\mathcal{R}_{\psi} \cap(\pi \times \pi)^{-1}(\mathcal{R})$ is compact and open.

Lemma 3.2. Suppose that $\mathcal{R}$ is an open subequivalence relation of $\mathcal{R}_{\varphi}$. Let $\mathcal{S}=\mathcal{R}_{\psi} \cap(\pi \times$ $\pi)^{-1}(\mathcal{R})$. If $B \subset X$ is a closed $\mathcal{R}$-étale thin subset, then $\pi^{-1}(B)$ is a closed $\mathcal{S}$-étale thin subset.

Proof. Let $\mu$ be an $\mathcal{S}$-invariant probability measure. Then we have

$$
\mu\left(\pi^{-1}(B)\right)=\pi_{*}(\mu)(B)=0,
$$

because $\pi_{*}(\mu)$ is a $\mathcal{R}$-invariant probability measure.

Take $\left(y, y^{\prime}\right) \in \mathcal{S}$. By the étaleness of $B$, we can find a clopen neighborhood $V$ of $\left(\pi(y), \pi\left(y^{\prime}\right)\right)$ in $\mathcal{R}$ such that, for $\left(x, x^{\prime}\right) \in V$, we have $x \in B$ if and only if $x^{\prime} \in B$. It is clear that $U=$ $\mathcal{R}_{\psi} \cap(\pi \times \pi)^{-1}(V)$ is a clopen neighborhood of $\left(y, y^{\prime}\right)$ in $\mathcal{S}$. Suppose $\left(z, z^{\prime}\right) \in U$. Because of $\left(\pi(z), \pi\left(z^{\prime}\right)\right) \in V$, we have

$$
z \in \pi^{-1}(B) \Leftrightarrow \pi(z) \in B \Leftrightarrow \pi\left(z^{\prime}\right) \in B \Leftrightarrow z^{\prime} \in \pi^{-1}(B) .
$$

It follows that $\pi^{-1}(B)$ is $\mathcal{S}$-étale.

Now we are ready to prove the main theorem.

Theorem 3.3. Let $\pi:(Y, \psi) \rightarrow(X, \varphi)$ be a factor map between Cantor minimal $\mathbb{Z}^{2}$-systems. If $(X, \varphi)$ is conjugate to a product of two Cantor minimal $\mathbb{Z}$-systems, then $\mathcal{R}_{\psi}$ is affable.

Proof. We may assume that $(X, \varphi)$ is equal to the product of two Cantor minimal $\mathbb{Z}$-systems $\left(X_{1}, \varphi_{1}\right)$ and $\left(X_{2}, \varphi_{2}\right)$, that is, $X=X_{1} \times X_{2}$ and $\varphi^{(n, m)}\left(x_{1}, x_{2}\right)=\left(\varphi_{1}^{n}\left(x_{1}\right), \varphi_{2}^{m}\left(x_{2}\right)\right)$ for all $(n, m) \in \mathbb{Z}^{2}$. Let $p=\left(p_{1}, p_{2}\right), U_{1}, U_{2}, B, B^{*}, \beta: B \rightarrow B^{*}$ and $\mathcal{R}^{\prime}$ be as in Theorem 2.10.

Put $\mathcal{S}=\mathcal{R}_{\psi} \cap(\pi \times \pi)^{-1}\left(\mathcal{R}^{\prime}\right)$. Thanks to Theorem 2.10 (1) and Lemma 3.1] the equivalence relation $\mathcal{S}$ is $\mathrm{AF}$, where the topology is given by $\mathcal{R}_{\psi}$. In order to show that $\mathcal{S}$ is minimal, let us choose $x_{i} \in X_{i} \backslash\left\{p_{i}\right\}$ and put $x_{0}=\left(x_{1}, x_{1}\right) \in X$. Take $y \in Y$ arbitrarily. The closure of $[\pi(y)]_{\mathcal{R}^{\prime}}$ is $X$, because $\mathcal{R}^{\prime}$ is minimal. It follows that the closure of $[y]_{\mathcal{S}}$ contains a preimage of $x_{0}$, namely $y_{0} \in Y$. On account of $\left[y_{0}\right]_{\mathcal{R}_{\psi}}=\left[y_{0}\right]_{\mathcal{S}}$, we can see that $\left[y_{0}\right]_{\mathcal{S}}$ is dense in $Y$. Therefore $[y]_{\mathcal{S}}$ is dense in $Y$.

Put $C=\pi^{-1}(B)$ and $C^{*}=\pi^{-1}\left(B^{*}\right)$. By means of Theorem 2.10 (2) and Lemma 3.2 we have that both $C$ and $C^{*}$ are closed $\mathcal{S}$-étale thin subsets. Moreover, it is easily seen that $\mathcal{S} \cap\left(C \times C^{*}\right)$ is empty.

We define a homeomorphism $\gamma: C \rightarrow C^{*}$ as follows. Take $y \in C$. If $\pi(y)=\left(p_{1}, x_{2}\right)$ for some $x_{2} \in U_{2}$, then we set $\gamma(y)=\psi^{(1,0)}(y)$. If $\pi(y)=\left(x_{1}, p_{2}\right)$ for some $x_{1} \in U_{1}$, then we set $\gamma(y)=\psi^{(0,1)}(y)$. It is routine to check that $\gamma$ is a well-defined homeomorphism from $C$ to $C^{*}$ and $\gamma$ induces an isomorphism between $\mathcal{S} \cap(C \times C)$ and $\mathcal{S} \cap\left(C^{*} \times C^{*}\right)$.

Let $\tilde{\mathcal{S}}$ be the equivalence relation generated by $\mathcal{S}$ and the graph of $\gamma$. We can apply the absorption theorem [GPS2, Theorem 4.18] to $\mathcal{S}$ and $\gamma: C \rightarrow C^{*}$ and get that $\tilde{\mathcal{S}}$ is affable.

The equivalence relation $\tilde{\mathcal{S}}$ is a little smaller than $\mathcal{R}_{\psi}$. We resolve this problem by using the absorption theorem three more times. Let $D_{1}=\pi^{-1}(p), D_{2}=\pi^{-1}\left(\varphi^{(0,1)}(p)\right), D_{3}=$ $\pi^{-1}\left(\varphi^{(1,1)}(p)\right)$ and $D_{4}=\pi^{-1}\left(\varphi^{(1,0)}(p)\right)$. At first, we apply the absorption theorem to $\psi^{(0,1)}$ : $D_{1} \rightarrow D_{2}$. Notice that

$$
\tilde{\mathcal{S}} \cap\left(D_{i} \times D_{i}\right)=\left\{(y, y): y \in D_{i}\right\}
$$


for each $i=1,2$ and that $\tilde{\mathcal{S}} \cap\left(D_{1} \times D_{2}\right)$ is empty. Therefore the hypothesis of the absorption theorem is trivially satisfied. It follows that the equivalence relation generated by $\tilde{\mathcal{S}}$ and

$$
\left\{\left(y, \psi^{(0,1)}(y)\right): y \in D_{1}\right\}
$$

is affable. Theorem 2.10 (5) and two more applications of the absorption theorem imply that $\mathcal{R}_{\psi}$ is affable.

\section{References}

[GPS1] Giordano, T.; Putnam, I. F.; Skau, C. F.; Topological orbit equivalence and $C^{*}$-crossed products, J. reine angew. Math. 469 (1995), 51-111.

[GPS2] Giordano, T.; Putnam, I. F.; Skau, C. F.; Affable equivalence relations and orbit structure of Cantor dynamical systems, Ergodic Theory Dynam. Systems 24 (2004), 441-475.

[GPS3] Giordano, T.; Putnam, I. F.; Skau, C. F.; The orbit structure of Cantor minimal $\mathbb{Z}^{2}$-systems, preprint.

[HPS] Herman, R. H.; Putnam, I. F.; Skau, C. F.; Ordered Bratteli diagrams, dimension groups and topological dynamics, Internat. J. Math. 3 (1992), 827-864.

e-mail:matui@math.s.chiba-u.ac.jp

Graduate School of Science and Technology,

Chiba University,

1-33 Yayoi-cho, Inage-ku,

Chiba 263-8522,

Japan. 